\title{
Lactobacillus rhamnosus GG Treatment Potentiates Intestinal Hypoxia-Inducible Factor, Promotes Intestinal Integrity and Ameliorates Alcohol-Induced
} Liver Injury

\author{
Yuhua Wang, ${ }^{* \dagger}$ Irina Kirpich, ${ }^{\dagger \S}$ Yanlong Liu, ${ }^{\dagger \ddagger}$ \\ Zhenhua Ma, ${ }^{\text {†ा }}$ Shirish Barve, ${ }^{+\S \|}$ \\ Craig J. McClain, ${ }^{\dagger \S \|_{\star \star \star}}$ and Wenke Feng ${ }^{\dagger \ddagger \S}$ \\ From the College of Food Science and Engineering, ${ }^{*}$ Jilin \\ Agricultural University, Changchun, China; the Departments of \\ Medicine $e^{\dagger}$ and Pharmacology and Toxicology" and the Alcohol \\ Research Center, ${ }^{\S}$ University of Louisville, Louisville, Kentucky; the \\ School of Pharmacy, ${ }^{\ddagger}$ Wenzhou Medical College, Zhejiang, \\ China; the First Hospital, ${ }^{\text {" }}$ Xi'an Jiaotong University, Shaanxi, \\ China; and the Robby Rex Veterans Affairs Medical Center,** \\ Louisville, Kentucky
}

Gut-derived endotoxin is a critical factor in the development and progression of alcoholic liver disease (ALD). Probiotics can treat alcohol-induced liver injury associated with gut leakiness and endotoxemia in animal models, as well as in human ALD; however, the mechanism or mechanisms of their beneficial action are not well defined. We hypothesized that alcohol impairs the adaptive response-induced hypoxia-inducible factor (HIF) and that probiotic supplementation could attenuate this impairment, restoring barrier function in a mouse model of ALD by increasing HIF-responsive proteins (eg, intestinal trefoil factor) and reversing established ALD. C57BJ/6N mice were fed the Lieber DeCarli diet containing 5\% alcohol for 8 weeks. Animals received Lactobacillus rbamnosus GG (LGG) supplementation in the last 2 weeks. LGG supplementation significantly reduced alcohol-induced endotoxemia and hepatic steatosis and improved liver function. LGG restored alcohol-induced reduction of HIF- $2 \alpha$ and intestinal trefoil factor levels. In vitro studies using the Caco- 2 cell culture model showed that the addition of LGG supernatant prevented alcohol-induced epithelial monolayer barrier dysfunction. Furthermore, gene silencing of HIF-1 $\alpha / 2 \alpha$ abolished the LGG effects, indicating that the protective effect of LGG is HIF-dependent. The present study provides a mechanistic insight for utilization of pro- biotics for the treatment of ALD, and suggests a critical role for intestinal hypoxia and decreased trefoil factor in the development of ALD. (Am J Pathol 2011, 179:2866-2875; DOI: 10.1016/j.ajpath.2011.08.039)

Alcohol consumption causes fatty liver, which can in some cases progress to inflammation, fibrosis, cirrhosis, and even liver cancer. ${ }^{1-5}$ The pathogenesis of alcoholic liver disease (ALD) is multifactorial. Previous studies showed that gutderived endotoxins contribute to ALD. Endotoxins derived from the cell wall of Gram-negative bacteria normally penetrate the gut epithelium in only trace amounts, because of intact intestinal barrier function; however, endotoxin leakiness may be increased under certain pathological conditions, such as chronic alcohol abuse. ${ }^{6}$

Although the exact mechanism by which endotoxins cause liver injury is still not clear, lipopolysaccharide stimulation of tumor necrosis factor- $\alpha$ and other inflammatory cytokines in ALD leading to liver injury is one likely pathway. Elimination of bacteria to prevent endotoxininduced liver injury has been used in clinical practice and experimental animal models. For example, the use of antibiotics to sterilize the gut to reduce endotoxin pro-

Supported by grants from the NIH (P01-AA017103, R01-AA015970, R01DK071765, R37-AA010762, R01-AA018016, R01-AA018869, P30AA019360, and RC2-AA019385 to C.J.M.), the Veterans Administration (C.J.M.), and the American Diabetes Association (07-07-JF-23 to W.F.). Support was also provided by funds from the China Scholarship Council (Y.W.), a grant from Jilin Provincial Government (20090576 to Y.W.), Wenzhou Medical College (Y.L.), and the Guanghua Foundation at Xi'an Jiaotong University (Z.M.)

Accepted for publication August 24, 2011.

Y.W. and I.K. contributed equally to the present work.

Supplemental material for this article can be found at $h t t p: / / a j p$. amjpathol.org or at doi: 10.1016/j.ajpath.2011.08.039.

Address reprint requests to Wenke Feng, Ph.D., 505 S. Hancock St., CTR517, Louisville, KY 40202, or Craig J. McClain, M.D., 505 S. Hancock St., CTR503, Louisville, KY 40202. E-mail: wenke.feng@louisville.edu or craig.mcclain@louisville.edu. 
duction prevented experimental alcohol-induced liver injury. ${ }^{7}$ Moreover, treatment with probiotics and prebiotics to alter the gut flora and reduce the Gram-negative bacteria population has been successfully used in several studies of alcohol-induced liver injury in rodents. ${ }^{8-10}$ We have recently reported that probiotic supplementation altered gut flora and improved liver function in human alcoholics. ${ }^{4}$ These studies strongly suggest that gut bacteria are a major factor in the pathogenesis of alcoholinduced liver injury and that endotoxin release in conjunction with impaired gut integrity may be one mechanism for activating proinflammatory pathways causing ALD.

The intestinal epithelium forms an essential barrier to gut luminal contents. The barrier function of intestinal epithelium is provided by paracellular apical junction complexes, including tight junctions and adherens junctions, ${ }^{11}$ located at the apical end of epithelial cells, and a thick mucus gel layer secreted by the intestinal mucosa. This structure provides a dynamic and regulated barrier to the flux of the luminal contents to the lamina propria.

The barrier function of the intestinal epithelium is regulated by the availability of oxygen. ${ }^{12-14}$ Intestinal epithelial cells function within a uniquely steep physiological oxygen gradient. Under stress conditions, the gradient shifts toward hypoxia, using more oxygen-independent glycolysis for energy production. This oxygen adaptation process is characterized by the expression of a master transcription factor, hypoxia-inducible factor (HIF). HIF is a heterodimer consisting of an $\alpha$-subunit and a $\beta$-subunit. ${ }^{15} \mathrm{HIF}-\beta$ is constitutively expressed and translocates into the nucleus, whereas stabilization and nuclear accumulation of HIF- $\alpha$ are induced by hypoxia or hypoxic mimics. Under normoxic conditions, the HIF- $\alpha$ subunit is degraded through a process mediated by hydroxylation of two proline residues in HIF- $\alpha$ through three HIF hydroxylases (prolyl hydroxylases). ${ }^{16-18}$ HIF activity increases the transcription of many genes that enable intestinal epithelial cells to be an effective barrier, ${ }^{13,19,20}$ and this HIF-dependent protection affects overall tissue integrity, rather than only tight junction proteins.

Probiotics are microorganisms that can alter the gut microbiota profile, resulting in improved barrier integrity. Lactobacillus rhamnosus Gorbach Goldin (LGG) is a widely studied probiotic. Although probiotics have several beneficial effects on intestinal function, including ameliorating diarrhea and prolonging remission in ulcerative colitis and pouchitis (these effects are generally attributed as anti-inflammatory and as reducing oxidative stress), the precise mechanisms by which probiotics attenuate alcohol-induced disruption of intestinal integrity and subsequent liver injury remain to be elucidated.

To determine whether LGG can attenuate established alcohol-induced intestinal barrier disruption, endotoxemia, and liver injury, we investigated the effect of LGG on epithelial cell permeability and severity of hepatic steatosis using in vivo (mouse) and in vitro (epithelial cell culture) models. We hypothesized that LGG would potentiate HIF function, increase epithelial protective gene expression, and preserve barrier function, thus reducing liver injury in the mouse model. We showed that LGG treatment in mice with established hepatic steatosis increases HIF-mediated signaling in intestinal epithelium, reduces endotoxemia, normalizes barrier function, and ameliorates alcohol-induced liver injury.

\section{Materials and Methods}

\section{Culture of $L$. rhamnosus $G G$}

LGG was purchased from the American Type Culture Collection (accession 53103; ATCC, Rockville, MD) and was cultured in Lactobacillus de Man, Rogosa, and Sharpe broth (Difco MRS broth; BD Biosciences-Advanced Bioprocessing, Sparks, MD) at $37^{\circ} \mathrm{C}$ in accordance with ATCC guidelines. Bacteria were harvested from MRS broth by centrifugation, and colony forming units (CFU) were counted by dilution and streaking on MRS agar plates (Difco) at $37^{\circ} \mathrm{C}$ overnight. To prepare supernatant, LGG culture broth was centrifuged and filtered through a $0.22-\mu \mathrm{m}$ filter. The supernatant was stored at $4^{\circ} \mathrm{C}$ for later use.

\section{Animal Studies}

Male C57BL/6N mice were obtained from Harlan Laboratories (Indianapolis, IN). Mice were pair-fed liquid diets (Lieber DeCarli) containing 17\% of energy as protein, $40 \%$ as corn oil, $7 \%$ as carbohydrate, and $35 \%$ as either alcohol (alcohol-fed, AF) or as an isocaloric maltosedextrin (pair-fed, PF) in the following groups: PF, AF, $A F+L G G$, and an overall control group of normal chow (13\% of energy from fat, N). LGG culture broth $\left(10^{9}\right.$ $\mathrm{CFU} /$ mouse per day) was added into the diet in last 2 weeks of the experiment. Mice were maintained on the treatments for a total of 8 weeks. At the end the of experiment, the mice were anesthetized with Avertin (2,2,2tribromoethanol) after overnight fasting. Plasma and tissue samples were collected for assays. All mice were treated according to the protocols reviewed and approved by the Institutional Animal Care and Use Committee of the University of Louisville.

\section{Liver and Intestine Histology}

Formalin-fixed, paraffin-embedded tissue sections were processed for staining with H\&E and then were studied by light microscopy.

\section{Caco-2 Monolayer Cell Culture and Barrier Function Analysis}

Caco-2 cells obtained from the ATCC were cultured in Eagle's minimal essential medium supplemented with $100 \mathrm{U} / \mathrm{mL}$ penicillin, $100 \mu \mathrm{g} / \mathrm{mL}$ streptomycin, and $10 \%$ fetal bovine serum at $37^{\circ} \mathrm{C}$ in a $5 \% \mathrm{CO}_{2}$ environment for 21 days. Culture medium was changed every 2 days. Caco-2 cells were subcultured after partial digestion with $0.25 \%$ trypsin-EDTA. For probiotic treatment, LGG cultural supernatant (LGG-s) from cultural broth at the density of $10^{9} \mathrm{CFU} / \mathrm{mL}$ was prepared and added into Caco-2 
Table 1. Primer Sequences for Real-Time RT-PCR

\begin{tabular}{|c|c|c|}
\hline Gene & Forward sequence & Reverse sequence \\
\hline TJP1 (alias ZO-1) & $5^{\prime}$ - АTCCCTCAACAAGGGCCATTC-3' & 5'-CACTTGTTTTGCCAGGTTTA-3' \\
\hline OCLN & $5^{\prime}$-CCAATGTGCAGGAGTGGG-3' & $5^{\prime}-$ CGCTGCTGTAACGAGGCT-3' \\
\hline CLDN1 & $5^{\prime}$-AAGTGCTTGGAAGACGATGA-3' & $5^{\prime}$-CTTGGTGTTGGGTAAAGAGGTT-3' \\
\hline MUC1 & $5^{\prime}-$ GTGCCCCCTAGCAGTACCG-3' & $5^{\prime}$-GACGTGCCCCTACAAGTTGG-3' \\
\hline MUC2 & $5^{\prime}$-ACTGCACATTCTTCAGCTGC-3' & $5^{\prime}$-ATTCATGAGGACGGTCTTGG-3' \\
\hline$C D 73$ & $5^{\prime}$-ATTGCAAAGTGGTTCAAAGTCA-3' & $5^{\prime}-$ ACACTTGGCCAGTAAAATAGGG-3' \\
\hline ITF & 5'-TGGTCCTGGCCTTGCTGT-3' & 5'-GGCACACTGGTTTGCAGACA-3' \\
\hline VEGF & 5'-TTACTGCTGTACCTCCACC-3' & $5^{\prime}$-ACAGGACGGCTTGAAGATG-3' \\
\hline HIF & $5^{\prime}-$ GCAAGCCCTGAAAGCG-3' & $5^{\prime}$-GGCTGTCCGACTTTGA-3' \\
\hline ACTB & $5^{\prime}-$ GAGACCTTCAACACCCC-3' & 5'-ATAGCTCTTCTCCAGGGAGG-3' \\
\hline
\end{tabular}

cell medium at $1 \%(\mathrm{v} / \mathrm{v}$; LGG-s/medium) concentration. Caco-2 cells grown on chamber slides (LabTek, Naperville, IL) were used for fluorescent staining of tight junction proteins ZO-1, occludin and claudin-1, whereas Caco-2 cells grown on 24-well plates were used for immunoblotting analysis. For measurement of epithelial barrier function, Caco-2 cells were seeded and cultured on 24-well inserts (pore size $0.4 \mu \mathrm{m}$; BD Biosciences, San Jose, CA) for 21 days, and then were treated with $5 \%$ ethanol in the presence or absence of the LGG-s for 24 hours before measurement. The transepithelial electrical resistance (TEER) of the filter-grown Caco-2 monolayers was measured with an epithelial volt ohmmeter (World Precision Instruments, Sarasota, FL). TEER was recorded with three consecutive measurements after subtracting the resistance value of the filters alone. For determination of paracellular permeability, fluorescein isothiocyanatedextran-4 (FD-4) was added to the apical compartment of Caco-2 cells at a concentration of $10 \mathrm{mg} / \mathrm{mL}$ in Eagle's minimal essential medium. After 90 minutes of incubation, the medium was collected and the FD-4 that penetrated to the medium was measured using a microplate fluorescence reader with an excitation wavelength of $485 \mathrm{~nm}$ and an emission wavelength of $530 \mathrm{~nm}$.

\section{Biochemical Assays}

Blood samples from control and alcohol-treated mice were drawn from the dorsal vena cava. Plasma was obtained by centrifuging the blood at $1560 \times \mathrm{g}$ for 30 minutes at $4^{\circ} \mathrm{C}$. Lipopolysaccharide levels were measured with a Limulus amebocyte lysate test kit (Lonza, Walkersville, MD) according to the manufacturer's instructions. Plasma alanine aminotransferase (ALT) was measured using an ALT Infinity enzymatic assay kit (Thermo Scientific, Waltham, MA). Liver tissue triglyceride, free fatty acid, and cholesterol concentrations were measured using Infinity kits (Thermo Scientific).

\section{Liver Triglyceride Assay}

Hepatic triglyceride levels were determined as described previously, ${ }^{4}$ using a triglyceride reagent (Thermo Fisher Scientific, Middletown, VA).

\section{Real-Time Quantitative RT-PCR Assay}

The mRNA levels were assessed by real-time quantitative RT-PCR. In brief, the total RNA was isolated with TRIzol reagent according to the manufacturer's protocol (Invitrogen, Carlsbad, CA) and was reverse-transcribed using a GeneAmp RNA PCR kit (Applied Biosystems, Foster City, CA). Primer sequences are given in Table 1. Realtime quantitative RT-PCR was performed on an ABI 7500 real-time PCR thermocycler with SYBR Green PCR master mix (Applied Biosystems). The relative quantities of target transcripts were calculated from duplicate samples after normalization of the data against the housekeeping gene $\beta$-actin. Dissociation curve analysis was performed after PCR amplification to confirm the specificity of the primers. Relative mRNA expression was calculated using the $\Delta \Delta \mathrm{C}_{\mathrm{T}}$ method. ${ }^{21}$

\section{RNA Interference}

SiRNAs targeting human HIF- $1 \alpha / 2 \alpha$ and a negative mismatched control were designed and synthesized by Ambion (Austin, TX). The Caco-2 monolayers cultured for 21 days were transfected with $100 \mathrm{nmol} / \mathrm{L} \mathrm{HIF-} 1 \alpha / 2 \alpha$ or negative mismatched siRNA using Lipofectamine 2000 transfection agent (Invitrogen) according to the manufacturer's instruction.

\section{Nuclear Extract Preparation}

Nuclear extracts were prepared as described previously, 22 with minor modifications. In brief, cells were washed once with ice-cold PBS. Ice-cold buffer (10 $\mathrm{mmol} / \mathrm{L}$ Tris- $\mathrm{HCl}, \mathrm{pH} 7.8,1.5 \mathrm{mmol} / \mathrm{L} \mathrm{MgCl}_{2}$, and 10 $\mathrm{mmol} / \mathrm{L} \mathrm{KCl}$ ) containing freshly added $0.4 \mathrm{mmol} / \mathrm{L}$ phenylmethylsulfonyl fluoride, $0.5 \mathrm{mmol} / \mathrm{L}$ dithiothreitol, and $1 \%$ protease inhibitor cocktail (Sigma-Aldrich, St. Louis, MO) was overlaid on cells in the well and incubated for 10 minutes. The cells were then harvested and lysed by Dounce homogenization. Nuclei were pelleted by centrifugation and then resuspended in ice-cold buffer $(20 \mathrm{mmol} / \mathrm{L}$ Tris- $\mathrm{HCl}, \mathrm{pH} 7.8,420 \mathrm{mmol} / \mathrm{L} \mathrm{KCl}, 1.5$ $\mathrm{mmol} / \mathrm{L} \mathrm{MgCl}_{2}$, and $20 \%$ glycerol) containing freshly added $0.4 \mathrm{mmol} / \mathrm{L}$ phenylmethylsulfonyl fluoride, 0.5 $\mathrm{mmol} / \mathrm{L}$ dithiothreitol, $1 \%$ protease inhibitor cocktail, and $1 \mathrm{mmol} / \mathrm{L} \mathrm{Na}_{3} \mathrm{VO}_{4}$ and incubated for 30 minutes on ice with occasional tapping. The extracts were clarified by 
centrifugation at $12,000 \times g$ for 15 minutes at $4^{\circ} \mathrm{C}$, placed in aliquots, and stored at $-80^{\circ} \mathrm{C}$.

\section{Immunoblotting Analysis}

Tissues were homogenized, and Caco-2 cell monolayers were lysed on ice for 30 minutes in radioimmunoprecipitation assay buffer $(50 \mathrm{mmol} / \mathrm{L}$ Tris $\cdot \mathrm{HCl}, \mathrm{pH} 7.4,150$ $\mathrm{mmol} / \mathrm{L} \mathrm{NaCl}, 2 \mathrm{mmol} / \mathrm{L}$ EDTA, $4 \mathrm{mmol} / \mathrm{L} \mathrm{Na}_{3} \mathrm{VO}_{4}, 40$ $\mathrm{mmol} / \mathrm{L} \mathrm{NaF}, 1 \%$ Triton $\mathrm{X}-100,1 \mathrm{mmol} / \mathrm{L}$ phenylmethylsulfonyl fluoride, $1 \%$ protease inhibitor cocktail) and centrifuged at $14,000 \times g$ for 10 minutes. The supernatant was collected. Aliquots of tissue and cell lysates and nuclear fractions prepared as above containing 10 to $30 \mu \mathrm{g}$ protein were loaded onto a $4 \%$ to $15 \%$ SDS-polyacrylamide gel. After electrophoresis, proteins were transferred to polyvinylidene fluoride or nitrocellular membrane. The membrane was probed with antibody against HIF- $1 \alpha$ (BD Biosciences-Advanced Bioprocessing), HIF-2 $\alpha$ (Novus, Littleton, CO), and ITF, VEGF, claudin-1, occludin, or $\beta$-actin (Santa Cruz Biotechnology, Santa Cruz, CA). The membrane was then processed with HRP-conjugated $\operatorname{lgG}$. The protein bands were visualized by an enhanced chemiluminescence detection system (GE Healthcare, Piscataway, NJ) and quantified by densitometry analysis.

\section{Immunofluorescence Microscopy of Tight-Junction Proteins}

Cryostat sections of the small intestines, and Caco-2 cells on chamber slides were fixed with cold methanol for 15 minutes at $-20^{\circ} \mathrm{C}$. They were then incubated with polyclonal rabbit anti-claudin-1, occludin, or ZO-1 antibodies (Zymed Laboratories, South San Francisco, CA) overnight at $4^{\circ} \mathrm{C}$, followed by incubation with a Cy3-conjugated antibody (Invitrogen) or fluorescein isothiocyanate-conjugated antibody (Invitrogen) for 30 minutes at room temperature.

\section{ROS Determination by Fluorescence Microscopy}

ROS accumulation in the small intestine and Caco-2 cells was examined by dihydroethidium fluorescence microscopy. ${ }^{23}$ Nonfluorescent dihydroethidium is oxidized by ROS to yield the red fluorescent product ethidium, which binds to nucleic acids, staining the nucleus a bright fluorescent red. Cryostat sections of ileum or Caco-2 cell chamber slides were incubated with $5 \mu \mathrm{mol} / \mathrm{L}$ dihydroethidium (Molecular Probes, Eugene, OR) for 30 minutes at $37^{\circ} \mathrm{C}$ in the dark. The ROS-catalyzed ethidium red fluorescence was examined under fluorescence microscopy. The relative fluorescence intensity was quantified with SigmaScan Pro 5 software (Systat Software, San Jose, CA).

\section{Statistical Analysis}

All data are expressed as means \pm SEM or as indicated. The data were analyzed by analysis of variance and Newman-Keuls multiple-comparison test. Differences between groups were considered significant at $P<0.05$.

\section{Results}

\section{LGG Supplementation Ameliorates Alcohol- Induced Hepatic Steatosis}

Alcohol exposure for 8 weeks produced a lower mouse body weight compared with the PF controls (Table 2). LGG supplementation in AF mice in the last 2 weeks did not affect body weight, compared with the AF mice without LGG. Alcohol exposure significantly increased plasma ALT level, and this elevation was attenuated by LGG supplementation. Plasma endotoxin level (lipopolysaccharide) was elevated in alcohol exposure group, and the rise was reduced in the LGG supplementation group. Alcohol exposure increased liver triglyceride, free fatty acid, and cholesterol levels. LGG supplementation significantly attenuated these increases. Alcohol exposure and LGG supplementation did not change the intestine/ body weight ratio (data not shown).

Representative photomicrographs depicting liver pathology (H\&E staining) are presented in Figure 1. Pairfeeding caused hepatic steatosis, compared with normal chow control, but no inflammation was observed after the 8-week feeding in the PF group. However, alcohol feeding increased hepatic damage, with necroinflammatory foci detectable microscopically. Two weeks of LGG supplementation in AF mice remarkably reduced the number and size of lipid droplets and inflammatory foci in the liver. Consistent with the serum ALT and liver triglyceride levels, LGG supplementation attenuated alcohol-induced liver pathology alterations, which include inflammatory cell infiltration and cell death.

Table 2. Characteristics and Biochemical Changes in LGG-Treated Mice

\begin{tabular}{lrrr}
\hline \multicolumn{1}{c}{ Characteristic } & \multicolumn{1}{c}{ PF } & AF & AF + LGG \\
\hline Body weight (g) & $28.1 \pm 0.64$ & $25.96 \pm 0.72^{\star}$ & $26.68 \pm 0.48$ \\
Plasma ALT (U/L) & $28.88 \pm 2.93$ & $43.66 \pm 2.02^{*}$ & $32.74 \pm 2.67^{\star \star}$ \\
Plasma LPS (mg/L) & $0.13 \pm 0.04$ & $0.37 \pm 0.17^{\star}$ & $0.17 \pm 0.04^{\star \star}$ \\
Liver TG (mg/dL) & $39.93 \pm 5.59$ & $100.2 \pm 8.17^{\star}$ & $89.7 \pm 3.87^{\star \star}$ \\
Free fatty acid (mE/g liver) & $0.14 \pm 0.04$ & $0.21 \pm 0.08^{\star}$ & $0.13 \pm 0.03$ \\
Cholesterol (mmol/g liver) & $12.75 \pm 0.78$ & $17.33 \pm 0.99^{\star}$ & $14.9 \pm 0.46$ \\
\hline
\end{tabular}

Values are reported as means \pm SD.

${ }^{\star} P<0.05$ versus $\mathrm{PA}$; ${ }^{* *} P<0.05$ versus $\mathrm{AF}$

AF, alcohol-fed; ALT, alanine aminotransferase; LGG, L. rhamnosus GG; LPS, lipopolysaccharide; PF, pair-fed; TG, triglyceride. 

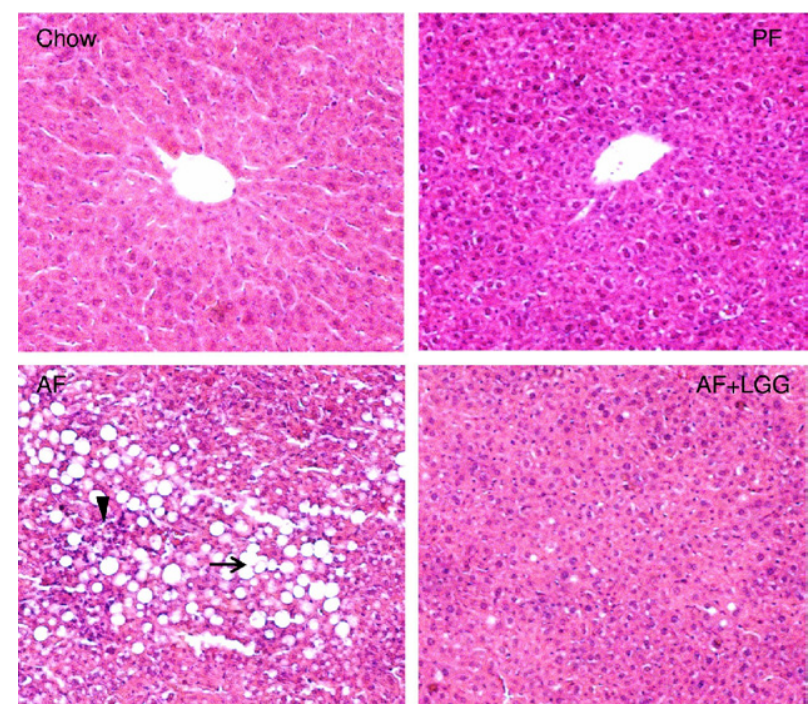

Figure 1. Effects of LGG supplementation on liver histology in mice chronically fed alcohol for 8 weeks. Light micrographs with H\&E staining reveal fat accumulation (arrow) and neutrophil infiltration (arrowhead) in the liver of alcohol-fed mice. Chow: normal chow; PF, pair-fed; AF, alcohol-fed; AF+LGG, alcohol-fed with LGG supplementation in the last 2 weeks. Magnification: $\times 200$

\section{LGG Treatment Increases ITF and VEGF}

\section{Expression}

We next sought to elucidate possible mechanisms for the observed protection by LGG in intestines and livers of the alcohol-treated mice by evaluating the effects of LGG on intestinal protective gene expression. Intestinal trefoil factor (ITF) and vascular endothelial growth factor (VEGF) play important roles in epithelial protection. Alcohol exposure caused significant reduction in ITF and VEGF protein levels in the ileum, which was normalized with LGG supplementation (Figure 2). Notably, expression of hypoxia-inducible factor $2 \alpha$ (HIF- $2 \alpha)$, which is an important transcription factor for ITF and VEGF, was almost
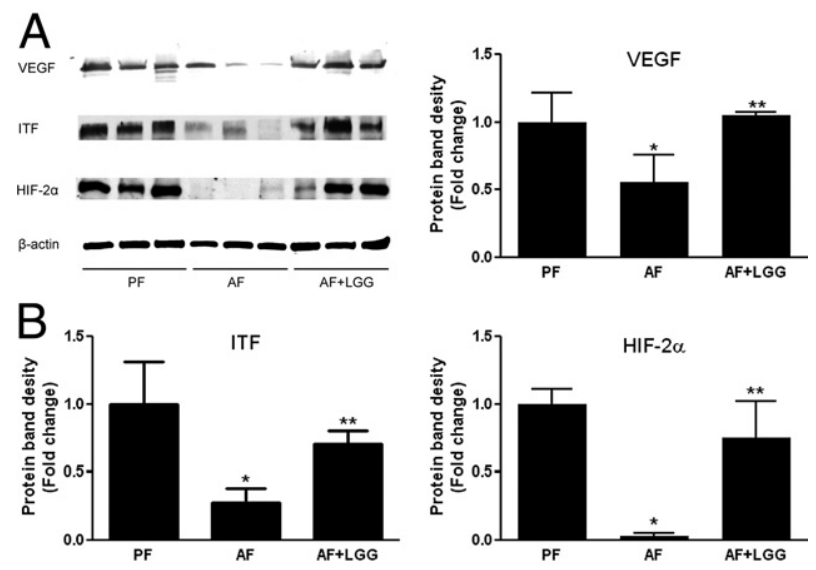

Figure 2. Effects of LGG supplementation on intestinal ITF, VEGF, and HIF- $2 \alpha$ protein and mRNA levels in mice chronically fed alcohol for 8 weeks. A: Western blot and protein band density quantitative analysis. The ratio to $\beta$-actin was calculated by setting the value of controls as 1 . B: mRNA levels were determined by real-time quantitative PCR. Results are expressed as means \pm SEM. ${ }^{*} P<0.05$ versus $P F ;{ }^{* *} P<0.05$ versus $A F$.

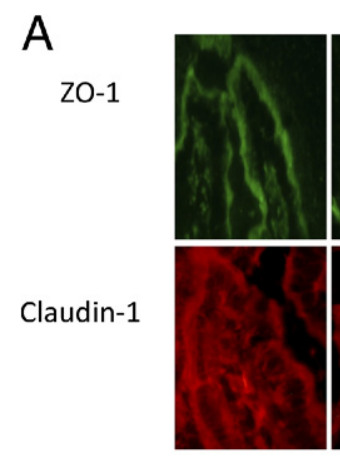

Normal Chow
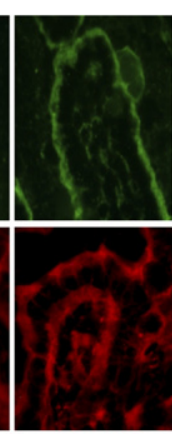

PF
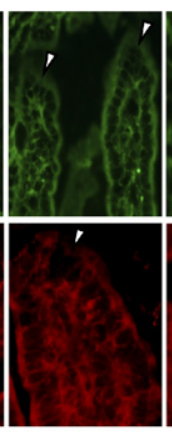

AF
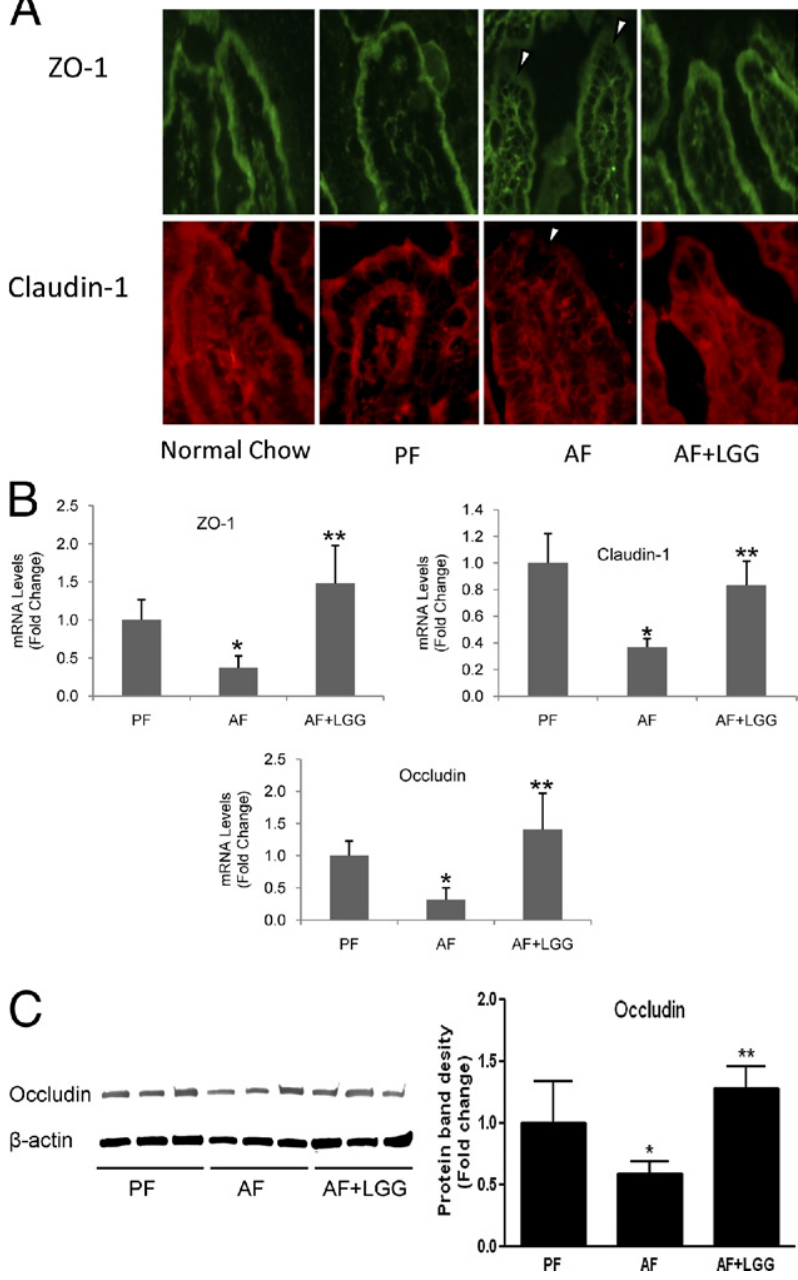

Figure 3. Effects of LGG supplementation on intestinal tight junctions in mice chronically fed alcohol for 8 weeks. A: Immunofluorescent microscopy detection of the ileal tight-junction proteins. Arrowheads indicate the disappearance of ZO- 1 and of claudin- 1 in AF mice. Magnification: $\times 200$ B: mRNA levels of ZO-1, claudin-1, and occludin. Results are means \pm SEM. ${ }^{*} P$ $<0.05$ versus $\mathrm{PF} ;{ }^{*} P<0.05$ versus $\mathrm{AF}$. C: Immunoblotting of and quantitative analysis of ileal occludin. The protein bands were quantified by densitometry analysis, normalized to $\beta$-actin.

completely abrogated by alcohol exposure, but LGG supplementation restored the HIF- $2 \alpha$ protein levels. The isoform HIF-1 $\alpha$, however, was not detected.

\section{LGG Supplementation Attenuates Alcohol- Induced Tight Junction Protein Expression}

Tight junction proteins play a critical role in gut permeability. Immunofluorescent staining revealed that alcohol exposure caused a reduction in distribution of both ZO-1 and claudin-1 between the adjacent epithelial cells in some parts of ileal epithelium, and LGG treatment normalized tight junction protein distribution (Figure $3 \mathrm{~A}$ ). Western blotting indicated a nonsignificant decrease in occludin protein level after alcohol exposure; LGG treatment attenuated this reduction (Figure $3 \mathrm{C}$ ). Alcohol exposure decreased but LGG supplementation increased the mRNA levels of ZO-1, claudin-1, and occludin (Figure 3B). 


\section{LGG Treatment Decreases Epithelial Cell Permeability in Caco-2 Cells}

To gain additional mechanistic insights, we used Caco-2 cells to evaluate the effect of LGG on intestinal epithelial integrity. Caco-2 cells are colon carcinoma cells that differentiate into intestine-like epithelial cells after 21 days of culture. Alcohol exposure significantly reduced the ITF protein level in Caco-2 cells. Because HIFs are transcription factors of ITF, we examined the protein levels of HIFs. $\mathrm{HIF}-1 \alpha$, and $\mathrm{HIF}-2 \alpha$ are barely detected under normoxic conditions, but alcohol decreased both HIF-1a and HIF-2a protein levels induced by hypoxia (see Supplemental Figure S1 at http://ajp.amjpathol.org). Alcohol exposure also reduced the tight junction proteins claudin-1 and occludin. LGG supernatant (LGG-s) treatment of alcohol-exposed cells increased these protein levels (Figure $4 \mathrm{~A}$ ). Alcohol treatment did not affect the mRNA levels of tight junction proteins, whereas a significant reduction of mRNA level of ITF by alcohol treatment was observed (Figure 4B). LGG-s treatment increased the gene expression of ITF and the tight junction proteins under both control and alcohol-treated conditions (Figure 4B). Immunofluorescent staining showed that alcohol exposure disrupted tight junction protein ZO-1, occludin, and claudin-1 distribution in Caco-2 cells, and LGG-s treatment prevented this effect (Figure 5A). We further analyzed the transcripts of other HIF-targeting genes in response to alcohol and LGG-s treatments. There were no changes in mRNA levels observed in alcohol-treated cells for genes involved in epithelial mucus protection [MUC1, MUC2,
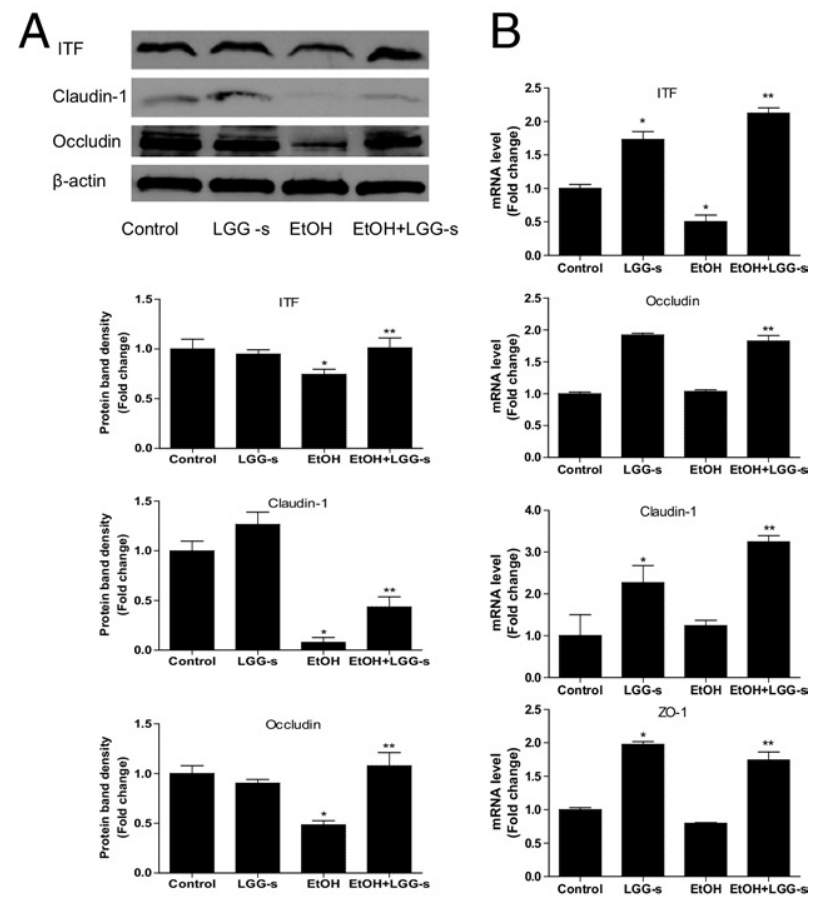

Figure 4. Effects of LGG on ITF and tight junctions in Caco-2 cells treated with 5\% alcohol for 24 hours. A: Western blotting and quantitative analysis of ITF, claudin-1, and occludin. B: mRNA levels of ITF, claudin-1, occludin, and ZO-1. ${ }^{*} P<0.05$ versus control; ${ }^{* *} P<0.05$ versus EtOH.
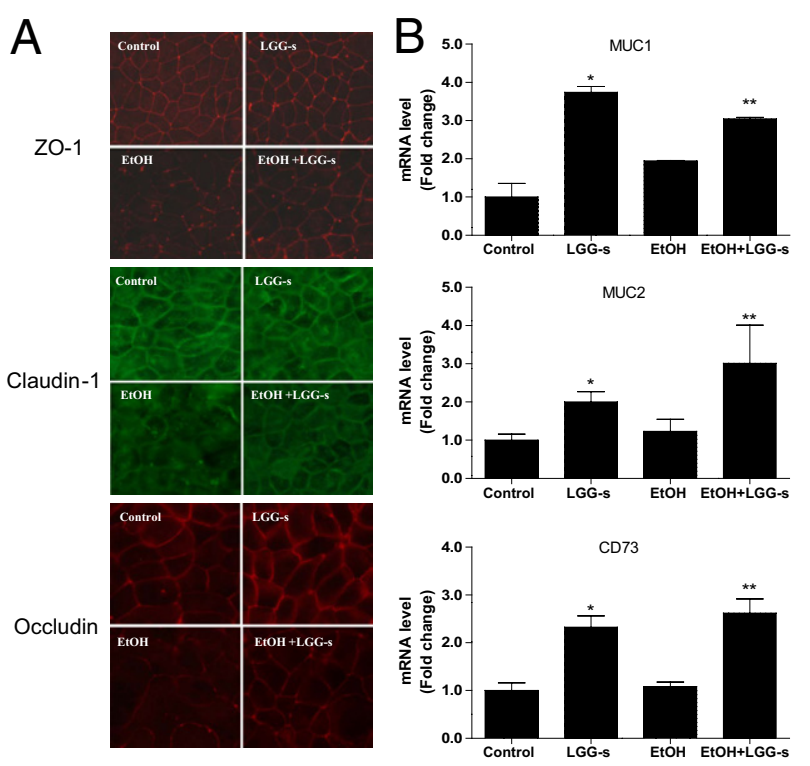

Figure 5. Effects of LGG on the protein distribution and mRNA levels of tight junction proteins and mucus protecting proteins in Caco- 2 cells treated with $5 \%$ alcohol for 24 hours. A: Immunofluorescent staining of ZO-1, occludin, and claudin-1. Magnification: $\times 400$ B: mRNA levels of Muc1, Muc-2, and CD73. ${ }^{*} P<0.05$ versus control; ${ }^{* *} P<0.05$ versus $\mathrm{EtOH}$.

and NT5E (alias CD73)], but LGG treatment increased the expression of these genes (Figure 5B).

Next, we investigated whether the alteration in barrierprotective proteins led to a change in epithelial permeability. Alcohol exposure caused a significant decrease in the epithelial TEER (Figure 6). Consistent with these results, the paracellular permeability to FD-4 was significantly increased by alcohol exposure. LGG-s treatment did not affect epithelial TEER and FD-4 measurements under control conditions, but normalized the alcohol-induced changes in TEER and FD-4.

\section{LGG Treatment Attenuates Alcohol-Induced ROS Formation in the lleum and in Caco-2 Cells}

Oxidative stress in the ileum and in Caco-2 cells was assessed by measuring ROS accumulation with ethidium fluorescence microscopy (Figure 7A) and image quantification (Figure 7B). Only trace amounts of ROS were detected in the ileum in the PF mice, but alcohol exposure caused ROS accumulation (as indicated by in-
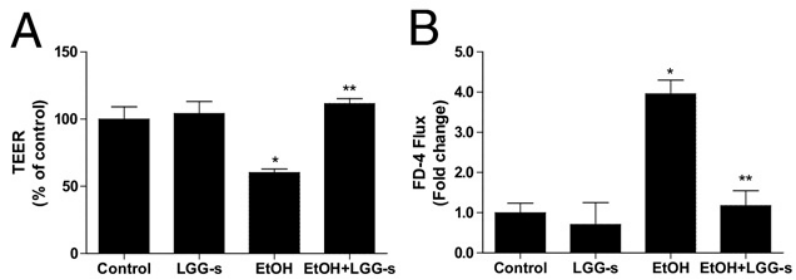

Figure 6. Effects of LGG on alcohol-induced changes in TEER and permeability in Caco-2 cell monolayers. TEER and permeability to FD-4 were assessed when Caco-2 cells had been grown for 21 days, allowing them to become fully differentiated. ${ }^{*} P<0.05$ versus control; ${ }^{* *} P<0.05$ versus EtOH 

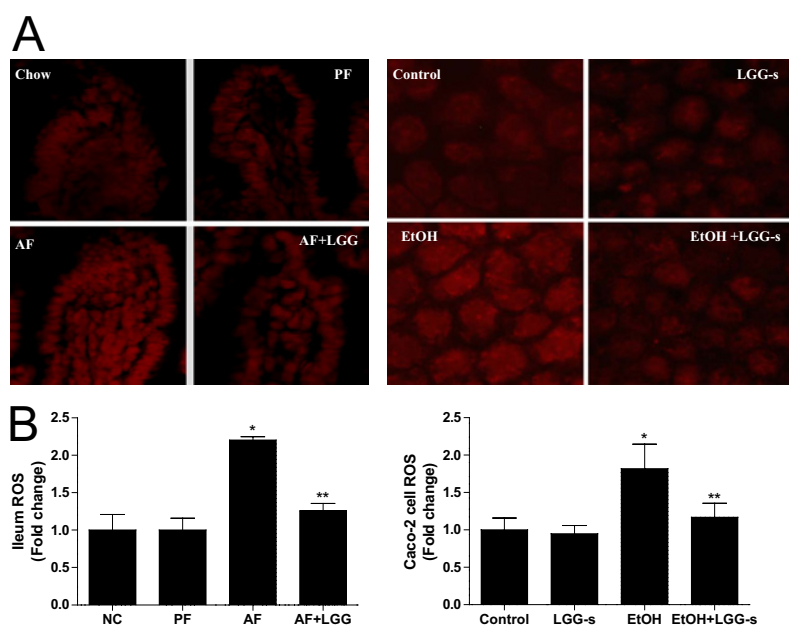

Figure 7. ROS accumulation in ileum and Caco-2 cells. A: Fluorescent micrographs of ROS in ileum of mice fed alcohol for 8 weeks (left, magnification $\times 200$ ) and in Caco- 2 cells treated with alcohol (right, magnification $\times 400$ ). Cryostat intestinal sections were incubated with dihydroethidium fluorescent dye at $5 \mu \mathrm{mol} / \mathrm{L}$, and red fluorescence was formed in the nuclei on oxidation by ROS. B: Quantitative analysis of the fluorescence intensity. ${ }^{*} P<0.05$ versus $P F ;{ }^{* *} P<0.05$ versus $A F$.

creased red fluorescence intensity; Figure 7). The alcohol-induced ROS formation was also detected in Caco-2 cells. LGG treatment attenuated the ROS accumulation in ileum and in Caco-2 cells.

\section{LGG Treatment Potentiates HIF Signaling in Caco-2 Cells}

As described above, HIF plays an important role in intestinal mucosal integrity, and the epithelial barrier-protective factors ITF, CD73, and VEGF, are transcriptional targets of HIF. We next determined whether LGG treatment alters HIF signaling. $\mathrm{CoCl}_{2}$, a hypoxia mimetic, induced an accumulation of $\mathrm{HIF}-1 \alpha$ and $\mathrm{HIF}-2 \alpha$ proteins, and this effect was attenuated by HIF- $1 \alpha / 2 \alpha$ siRNA treatment. As expected, depleting HIF- $1 \alpha$ and HIF-2 $\alpha$ genes decreased ITF protein level, and LGG-s was not able to reverse this effect (Figure $8 \mathrm{~A}$ ). In addition, we found that silencing HIF-1/2 $\alpha$ decreased tight junction occludin and claudin-1 protein levels in Caco-2 cells under normoxic and hypoxic conditions (see Supplemental Figure S2 at http://ajp.amjpathol.org). Importantly, down-regulation of HIF signaling by siRNA completely abolished the LGGs-conferred protection against alcohol-induced reduction in TEER and increase in FD-4 measurements (Figure 8B). These results indicate that probiotic protection of epithelial barrier integrity is HIF-dependent.

\section{Discussion}

The present investigation of the effects of the LGG supplementation on alcohol-induced intestinal epithelial cell permeability, endotoxemia, liver triglyceride accumulation, and hepatic injury revealed two major findings. First, 2-week LGG supplementation showed beneficial effects on liver function in mice chronically fed an alcohol Lieber
DeCarli diet. Second, this beneficial effect of LGG was associated with an improvement in HIF signaling, leading to the up-regulation of important intestinal barrier-protective genes and subsequent protection against the alcohol-induced endotoxemia and liver injury. These findings indicate that activation of HIF signaling plays a critical role in the beneficial effects of probiotic treatment of alcoholic liver disease.

Our findings confirm previous studies showing that probiotic supplementation improved alcohol-induced liver injury, and that alcohol-induced endotoxemia and epithelium leakiness is a potential mechanism for liver injury. ${ }^{8,24,25}$ In chronic alcohol-related models of liver injury in mice or rats, probiotic supplementation significantly lowered alcohol-induced endotoxemia and liver damage. ${ }^{8}$ Probiotics also prevented endotoxemia and liver damage in an acute alcohol exposure model. ${ }^{25}$ Our studies are unique in that we treated animals that had already been consuming alcohol for 6 weeks; this was a treatment rather than a prevention study. This simulates the human situation, and so is different from almost all rodent ALD studies (which involve prevention).

LGG protection against intestinal barrier dysfunction has been demonstrated in a wide range of pathological conditions; these include gastric hyperpermeability in acute alcohol exposure, increased intestinal permeability caused by cow's milk in suckling rats, ${ }^{26}$ increased intestinal permeability and inflammation in IL-10 knockout mice, ${ }^{27,28}$ and psychological stress-induced intestinal dysfunction. .9-31 $^{29}$ Our previous studies showed that chronic alcohol exposure damaged intestinal tight junctions and caused intestinal hyperpermeability, subsequently resulting in lipopolysaccharide absorption and eventually causing liver injury in a mouse model of ALD. ${ }^{32,33}$ We also showed that probiotic supplementation in patients with alcohol-induced liver injury improved bowel flora and liver enzymes. ${ }^{4}$ Thus, our own studies and those of others suggest that probiotics, including LGG, can improve or normalize intestinal barrier function and attenuate alcohol-induced liver injury.
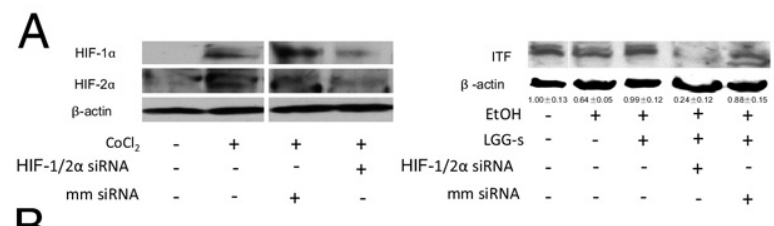

B
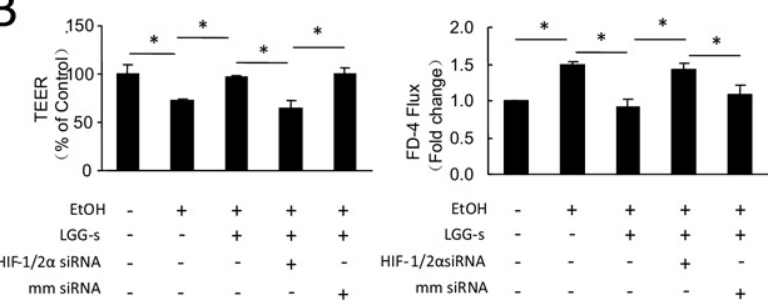

Figure 8. Effects of HIF- $1 / 2 \alpha$ siRNA on epithelial permeability in Caco-2 cells treated with LGG and alcohol. Caco- 2 cells were transfected with human HIF- $1 / 2 \alpha$ siRNAs or control siRNA on Day 21 for 48 hours, and then treated with $200 \mu \mathrm{mol} / \mathrm{L} \mathrm{CoCl}_{2}$ for 24 hours to induce HIF- $\alpha$ accumulation. A: Immunoblot analysis of HIF- $1 \alpha$, HIF- $2 \alpha$, and ITF protein levels. B: Measurements of TEER and FD-4 permeability. ${ }^{*} P<0.05$. 
The mechanisms underlying the protective or therapeutic effects on barrier function and alcohol-induced liver injury are not fully defined. Probiotics, including $L G G$, have been shown to inhibit inflammatory signaling in experimental colitis. ${ }^{34,35}$ Probiotics may also directly modulate immune cell functions, including dendritic cells, macrophages, and natural killer T cells. ${ }^{36,37} \mathrm{~A}$ recent study by Forsyth et $\mathrm{al}^{8}$ showed that probiotics inhibit inflammatory signaling via NF- $\kappa \mathrm{B}$ activation, which has been identified as a critical pathway in alcohol-induced oxidative stress and intestinal hyperpermeability. However, it is unclear exactly how probiotics preserve or restore the intestinal integrity.

In the present study, we showed that ITF and VEGF were significantly reduced in alcohol-treated ileum. VEGF is a proangiogenic growth factor that has protective effects on intestinal epithelial apoptosis. ${ }^{38}$ ITF is a peptide produced by intestinal goblet cells that modifies mucin and plays a critical role in the intestinal mucus protection. ${ }^{39}$ In comparison with other organs of the body, intestinal epithelium experiences a uniquely steep physiological oxygen gradient. ${ }^{13,40}$ Under pathological conditions, increased tissue metabolism renders the inflamed mucosa and epithelium hypoxic, which activates the transcription factor HIF as a compensatory mechanism. Studies have shown that genetic loss of epithelial HIF- $1 \alpha$ expression resulted in a more severe colitic phenotype than that found in wild-type animals, and constitutively overexpressing intestinal epithelial HIF-1 was protective by increasing ITF, multidrug resistance gene-1, and CD73. ${ }^{20}$ In general, epithelial hypoxia (physiological and pathological) has a profound effect on intestinal energy metabolism and barrier function. Studies of the hypoxia-elicited pathways have shown a dependence on HIF-mediated transcriptional responses, and the functional proteins encoded by HIF-dependent mRNAs localize primarily to the most luminal aspect of polarized epithelia. ${ }^{19,41,42} \mathrm{HIF}$-dependent protective factors include increased mucin production by molecules that modify mucins, such as ITF ${ }^{19}$; xenobiotic clearance by P-glycoprotein ${ }^{41}$; nucleotide metabolism by $5^{\prime}$-nucleotidases, such as $\mathrm{CD}^{4} 3^{42}$; and nucleotide signaling through the adenosine $\mathrm{A}_{2 \mathrm{~B}}$ receptor. ${ }^{43}$ However, these HIF-mediated protective mechanisms can be disrupted under certain pathogenic conditions. Indeed, in a large-scale gene profiling study by our research group, ITF was dramatically down-regulated (>50-fold) in the livers of alcoholfed rodents (an observation that, in part, prompted the present study). ${ }^{44,45}$ Consistent with this concept, the present data show that chronic alcohol exposure reduces HIF- $2 \alpha$ expression in the ileum, and that LGG supplementation was able to restore HIF- $2 \alpha$ and the barrierprotective factor ITF.

The protective effects of LGG on alcoholic intestinal cells appear to be HIF-dependent. In inflammatory bowel disease, disruption of the HIF-1 gene resulted in more severe colitis, whereas overexpression of HIF-1 was protective. ${ }^{20}$ Furthermore, inactivation of HIF prolyl hydroxylase, which hydroxylizes HIF- $\alpha$ (leading to its degradation), protects the epithelial barrier. The present findings show that silencing HIF-1/2 $\alpha$ by siRNA abolished the protective effect of LGG on alcohol-induced permeability in a Caco-2 cell monolayer, indicating the requirement of HIF for the functioning of $L G G$.

Oxidative stress plays a critical role in alcohol-induced intestinal barrier function. Alcohol exposure induces oxidative stress in the intestine, and anti-oxidant treatments prevent gut leakiness. ${ }^{8,46-48}$ Alcohol exposure induced a progressive disruption of ZO-1 from the tight junction. ${ }^{49}$ Our previous studies have shown that treatment with $N$ acetyl-cysteine attenuates ROS formation and epithelial barrier dysfunction in Caco-2 cells. ${ }^{33}$ These studies suggest that oxidative stress-mediated epithelial barrier function involves disruption of tight junction in alcoholexposed intestinal epithelial cells. Our data support the notion that LGG treatment results in a decrease in oxidative stress, which leads to improved tight junction protein expression and distribution and thus to restoration of barrier function.

Although live probiotics produce beneficial effects on intestinal integrity, the protective effect of heat-inactivated probiotics or probiotic-produced nonviable soluble proteins is more controversial. Recent studies by Polk and colleagues ${ }^{50,51}$ showed that soluble proteins produced by LGG regulate intestinal epithelial cell survival and growth, suggesting that the components secreted by LGG may be useful to prevent intestinal injury. Specifically, two purified proteins (p75 and p40 from LGG culture supernatant) protect intestinal epithelial cells from apoptosis, promote proliferation, and activate Akt in a PI3K-dependent manner in both cell and organ culture models. ${ }^{50,51}$ In addition, studies have indicated several potential mechanisms under various pathological conditions. Secreted bioactive factors from several probiotic bacteria contributed to epithelial integrity ${ }^{52}$ by promoting epithelial cell growth and inhibited cytokine-induced epithelial cell apoptosis via the Akt pathway. ${ }^{50,52}$ Culture

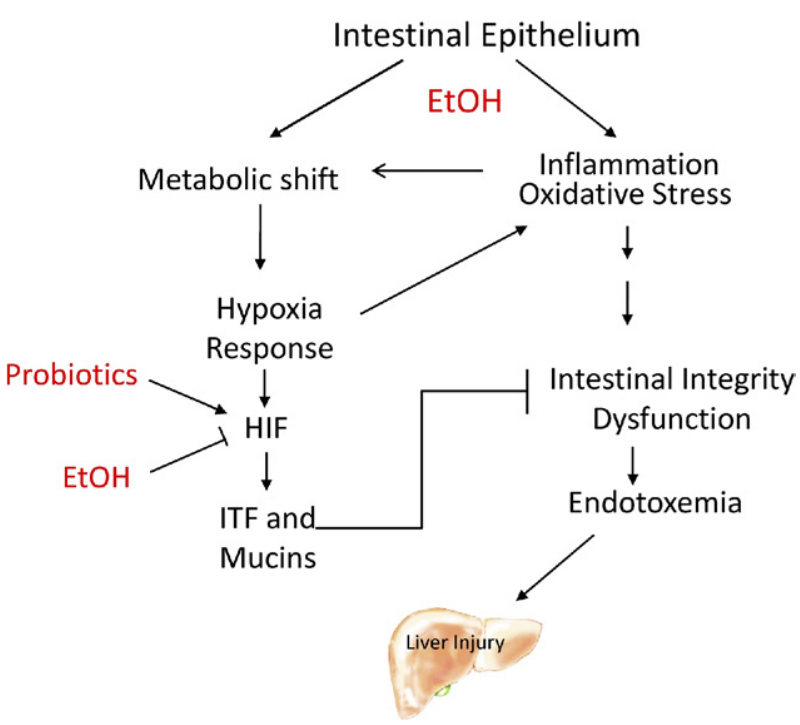

Figure 9. Schematic illustration of the mechanisms of alcohol-induced liver injury and its amelioration by probiotics. Alcohol (EtOh) impairs epithelial integrity and probiotic preserves the barrier function by maintaining HIF activity and mucus molecules, and consequently, reduces endotoxin release and protects liver from alcohol-induced injury. 
supernatant from the probiotic VSL\#3 enhanced epithelial barrier function through inhibition of NF- $\mathrm{B}$ B activity and expression of heat-shock proteins. ${ }^{52,53}$ Some conjugated linoleic acids secreted from probiotics are antiinflammatory in epithelial cells. ${ }^{54}$ The present study confirmed the findings that LGG-secreted molecules were protective in Caco-2 cells. LGG-s incubation attenuated both the alcohol-induced the reduction in epithelial resistance and the increase in permeability. However, the protective effects by nonviable secreted proteins are likely probiotic-specific. For example, an $L$. casei culture supernatant failed to provide a protection against inflammatory cytokine-induced epithelial barrier dysfunction. ${ }^{55}$ These varying results suggest that individual probiotics may produce varying secreted compounds that have different properties of action on epithelial integrity. In addition, alcohol and inflammatory cytokines may exert separate deleterious effects on the intestinal epithelial barrier.

Based on the present findings, we propose the following scheme of events (Figure 9). In addition to physiological intestinal hypoxia, alcohol induces pathological hypoxia in the intestine, which damages intestinal epithelial function. The normal epithelial adaptive response to hypoxia (ie, up-regulation of HIF- $\alpha$ ) is impaired by alcohol exposure. As a consequence, barrier-protective factors, such as ITF, are decreased, leading to increased epithelial permeability, endotoxin release, and eventual liver injury. Probiotic treatment increases HIF- $\alpha$ and its targeted barrier-protective factors, preserves intestinal integrity, decreases permeability, and protects the liver from alcohol injury.

In summary, the present study shows that supplementation of LGG in alcohol-exposed mice attenuates alcohol-induced gut-derived endotoxemia, hepatic lipid accumulation, and liver injury. Our data suggest that HIF signaling plays a critical role in the protective effect of probiotic administration in ALD by regulating HIF-targeted epithelial barrier-protective factors. The present findings provide novel scientific evidence for therapeutic intervention with probiotics in ALD. These data also provide a rationale for investigating probiotic and intestine trefoil factor effects in other alcohol-gut interaction, such as those in HIV-infected persons abusing alcohol.

\section{Acknowledgments}

We thank Keith C. Falkner and Zhanxiang Zhou for helpful discussion, Li Zhan for technical support, and Marion McClain for manuscript proofreading.

\section{References}

1. Beier JI, McClain CJ: Mechanisms and cell signaling in alcoholic liver disease. Biol Chem 2010, 391:1249-1264

2. Frazier TH, Stocker AM, Kershner NA, Marsano LS, McClain CJ: Treatment of alcoholic liver disease. Therap Adv Gastroenterol 2011, 4:63-81

3. McClain CJ, Song Z, Barve SS, Hill DB, Deaciuc I: Recent advances in alcoholic liver disease. IV. Dysregulated cytokine metabolism in alcoholic liver disease. Am J Physiol Gastrointest Liver Physiol 2004, 287:G497-G502

4. Kirpich IA, Solovieva NV, Leikhter SN, Shidakova NA, Lebedeva OV, Sidorov PI, Bazhukova TA, Soloviev AG, Barve SS, McClain CJ, Cave $\mathrm{M}$ : Probiotics restore bowel flora and improve liver enzymes in human alcohol-induced liver injury: a pilot study. Alcohol 2008, 42:675-682

5. Rao RK: Acetaldehyde-induced barrier disruption and paracellular permeability in Caco-2 cell monolayer. Methods Mol Biol 2008, 447: 171-183

6. Parlesak A, Schafer C, Schutz T, Bode JC, Bode C: Increased intestinal permeability to macromolecules and endotoxemia in patients with chronic alcohol abuse in different stages of alcohol-induced liver disease. J Hepatol 2000, 32:742-747

7. Adachi Y, Moore LE, Bradford BU, Gao W, Thurman RG: Antibiotics prevent liver injury in rats following long-term exposure to ethanol. Gastroenterology 1995, 108:218-224

8. Forsyth CB, Farhadi A, Jakate SM, Tang Y, Shaikh M, Keshavarzian A: Lactobacillus GG treatment ameliorates alcohol-induced intestinal oxidative stress, gut leakiness, and liver injury in a rat model of alcoholic steatohepatitis. Alcohol 2009, 43:163-172

9. Yan AW, Fouts E, Brandl J, Starkel P, Torralba M, Schott E, Tsukamoto $\mathrm{H}$, Nelson E, Brenner A, Schnabl B: Enteric dysbiosis associated with a mouse model of alcoholic liver disease. Hepatology 2011, 53:96105

10. Mutlu E, Keshavarzian A, Engen P, Forsyth CB, Sikaroodi M, Gillevet $P$ : Intestinal dysbiosis: a possible mechanism of alcohol-induced endotoxemia and alcoholic steatohepatitis in rats. Alcohol Clin Exp Res 2009, 33:1836-1846

11. Hartsock A, Nelson WJ: Adherens and tight junctions: structure, function and connections to the actin cytoskeleton. Biochim Biophys Acta 2008, 1778:660-669

12. Laukoetter MG, Bruewer M, Nusrat $A$ : Regulation of the intestinal epithelial barrier by the apical junctional complex. Curr Opin Gastroenterol 2006, 22:85-89

13. Taylor CT, Colgan SP: Hypoxia and gastrointestinal disease. J Mol Med 2007, 85:1295-1300

14. Turner JR: Intestinal mucosal barrier function in health and disease Nat Rev Immunol 2009, 9:799-809

15. Wang GL, Jiang BH, Rue EA, Semenza GL: Hypoxia-inducible factor 1 is a basic-helix-loop-helix-PAS heterodimer regulated by cellular $\mathrm{O} 2$ tension. Proc Natl Acad Sci USA 1995, 92:5510-5514

16. Kaelin WG Jr, Ratcliffe PJ: Oxygen sensing by metazoans: the central role of the HIF hydroxylase pathway. Mol Cell 2008, 30:393-402

17. Semenza G: Signal transduction to hypoxia-inducible factor 1. Biochem Pharmacol 2002, 64:993-998

18. Semenza GL: Hypoxia-inducible factor 1 (HIF-1) pathway. Sci STKE 2007, 2007(407):cm8

19. Furuta GT, Turner JR, Taylor CT, Hershberg RM, Comerford K, Narravula S, Podolsky DK, Colgan SP: Hypoxia-inducible factor 1-dependent induction of intestinal trefoil factor protects barrier function during hypoxia. J Exp Med 2001, 193:1027-1034

20. Karhausen J, Furuta GT, Tomaszewski JE, Johnson RS, Colgan SP, Haase VH: Epithelial hypoxia-inducible factor-1 is protective in murine experimental colitis. J Clin Invest 2004, 114:1098-1106

21. Livak KJ, Schmittgen TD: Analysis of relative gene expression data using real-time quantitative PCR and the 2(-Delta Delta C(T)) method. Methods 2001, 25:402-408

22. Feng W, Ye F, Xue W, Zhou Z, Kang YJ: Copper regulation of hypoxia-inducible factor-1 activity. Mol Pharmacol 2009, 75:174-182

23. Xue W, Liu Q, Cai L, Wang Z, Feng W: Stable overexpression of human metallothionein-IIA in a heart-derived cell line confers oxidative protection. Toxicol Lett 2009, 188:70-76

24. Nanji AA, Khettry U, Sadrzadeh SM: Lactobacillus feeding reduces endotoxemia and severity of experimental alcoholic liver (disease). Proc Soc Exp Biol Med 1994, 205:243-247

25. Marotta F, Barreto R, Wu CC, Naito Y, Gelosa F, Lorenzetti A, Yoshioka M, Fesce E: Experimental acute alcohol pancreatitis-related liver damage and endotoxemia: synbiotics but not metronidazole have a protective effect. Chin J Dig Dis 2005, 6:193-197

26. Isolauri E, Majamaa H, Arvola T, Rantala I, Virtanen E, Arvilommi H: Lactobacillus casei strain GG reverses increased intestinal permeability induced by cow milk in suckling rats. Gastroenterology 1993 , 105:1643-1650 
27. Madsen KL, Doyle JS, Jewell LD, Tavernini MM, Fedorak RN: Lactobacillus species prevents colitis in interleukin 10 gene-deficient mice. Gastroenterology 1999, 116:1107-1114

28. Schultz M, Veltkamp C, Dieleman LA, Grenther WB, Wyrick PB, Tonkonogy SL, Sartor RB: Lactobacillus plantarum 299V in the treatment and prevention of spontaneous colitis in interleukin-10-deficient mice. Inflamm Bowel Dis 2002, 8:71-80

29. Eutamene H, Lamine F, Chabo C, Theodorou V, Rochat F, Bergonzell GE, Corthesy-Theulaz I, Fioramonti J, Bueno L: Synergy between Lactobacillus paracasei and its bacterial products to counteract stress-induced gut permeability and sensitivity increase in rats. J Nutr 2007, 137:1901-1907

30. Eutamene $\mathrm{H}$, Bueno L: Role of probiotics in correcting abnormalities of colonic flora induced by stress. Gut 2007, 56:1495-1497

31. Zareie M, Johnson-Henry K, Jury J, Yang PC, Ngan BY, McKay DM, Soderholm JD, Perdue MH, Sherman PM: Probiotics prevent bacteria translocation and improve intestinal barrier function in rats following chronic psychological stress. Gut 2006, 55:1553-1560

32. Zhong W, Zhao Y, McClain CJ, Kang YJ, Zhou Z: Inactivation of hepatocyte nuclear factor-4\{alpha\} mediates alcohol-induced downregulation of intestinal tight junction proteins. Am J Physiol Gastrointest Liver Physiol 2010, 299:G643-G651

33. Zhong W, McClain CJ, Cave M, Kang YJ, Zhou Z: The role of zinc deficiency in alcohol-induced intestinal barrier dysfunction. Am J Physiol Gastrointest Liver Physiol 2010, 298:G625-G633

34. Ewaschuk JB, Dieleman LA: Probiotics and prebiotics in chronic inflammatory bowel diseases. World J Gastroenterol 2006, 12:59415950

35. Miyauchi E, Morita H, Tanabe S: Lactobacillus rhamnosus alleviates intestinal barrier dysfunction in part by increasing expression of zonula occludens-1 and myosin light-chain kinase in vivo. J Dairy Sci 2009, 92:2400-2408

36. Feleszko W, Jaworska J, Rha RD, Steinhausen S, Avagyan A, Jaudszus A, Ahrens B, Groneberg DA, Wahn U, Hamelmann E: Probioticinduced suppression of allergic sensitization and airway inflammation is associated with an increase of $T$ regulatory-dependent mechanisms in a murine model of asthma. Clin Exp Allergy 2007, 37:498505

37. Ma X, Hua J, Li Z: Probiotics improve high fat diet-induced hepatic steatosis and insulin resistance by increasing hepatic NKT cells. J Hepatol 2008, 49:821-830

38. Nakajima T, Ueda T, Takeyama Y, Yasuda T, Shinzeki M, Sawa H, Kuroda Y: Protective effects of vascular endothelial growth factor on intestinal epithelial apoptosis and bacterial translocation in experimental severe acute pancreatitis. Pancreas 2007, 34:410-416

39. Kjellev S: The trefoil factor family-small peptides with multiple functionalities. Cell Mol Life Sci 2009, 66:1350-1369

40. Colgan SP, Taylor CT: Hypoxia: an alarm signal during intestinal inflammation. Nat Rev Gastroenterol Hepatol 2010, 7:281-287

41. Comerford KM, Wallace TJ, Karhausen J, Louis NA, Montalto MC Colgan SP: Hypoxia-inducible factor-1-dependent regulation of the multidrug resistance (MDR1) gene. Cancer Res 2002, 62:3387-3394

42. Synnestvedt K, Furuta GT, Comerford KM, Louis N, Karhausen J, Eltzschig HK, Hansen KR, Thompson LF, Colgan SP: Ecto-5'-nucle- otidase (CD73) regulation by hypoxia-inducible factor-1 mediates permeability changes in intestinal epithelia. J Clin Invest 2002 , 110:993-1002

43. Eltzschig HK, Ibla JC, Furuta GT, Leonard MO, Jacobson KA, Enjyoji K, Robson SC, Colgan SP: Coordinated adenine nucleotide phosphohydrolysis and nucleoside signaling in posthypoxic endothelium: role of ectonucleotidases and adenosine A2B receptors. J Exp Med 2003 , 198:783-796

44. Deaciuc IV, Song Z, McClain CJ: Lessons from large-scale gene profiling of the liver in alcoholic liver disease. Hepatol Res 2005, 31:187-192

45. Deaciuc IV, Doherty DE, Burikhanov R, Lee EY, Stromberg AJ, Peng $X$, DE Villiers WJ: Large-scale gene profiling of the liver in a mouse model of chronic, intragastric ethanol infusion. J Hepatol 2004, 40: 219-227

46. Bagchi D, Carryl OR, Tran MX, Krohn RL, Bagchi DJ, Garg A, Bagchi M, Mitra S, Stohs SJ: Stress, diet and alcohol-induced oxidative gastrointestinal mucosal injury in rats and protection by bismuth subsalicylate. J Appl Toxicol 1998, 18:3-13

47. Keshavarzian A, Choudhary S, Holmes EW, Yong S, Banan A, Jakate S, Fields JZ: Preventing gut leakiness by oats supplementation ameliorates alcohol-induced liver damage in rats. J Pharmacol Exp Ther 2001, 299:442-448

48. Keshavarzian A, Farhadi A, Forsyth CB, Rangan J, Jakate S, Shaikh M, Banan A, Fields JZ: Evidence that chronic alcohol exposure promotes intestinal oxidative stress, intestinal hyperpermeability and endotoxemia prior to development of alcoholic steatohepatitis in rats. J Hepatol 2009, 50:538-547

49. Ma TY, Nguyen D, Bui V, Nguyen H, Hoa N: Ethanol modulation of intestinal epithelial tight junction barrier. Am J Physiol 1999, 276 : G965-G974

50. Yan F, Cao H, Cover TL, Whitehead R, Washington MK, Polk DB: Soluble proteins produced by probiotic bacteria regulate intestinal epithelial cell survival and growth. Gastroenterology 2007, 132:562575

51. Seth A, Yan F, Polk DB, Rao RK: Probiotics ameliorate the hydrogen peroxide-induced epithelial barrier disruption by a PKC- and MAP kinase-dependent mechanism. Am J Physiol Gastrointest Liver Physiol 2008, 294:G1060-G1069

52. Ohland CL, Macnaughton WK: Probiotic bacteria and intestinal epithelial barrier function. Am J Physiol Gastrointest Liver Physiol 2010, 298:G807-G819

53. Petrof EO, Kojima K, Ropeleski MJ, Musch MW, Tao Y, De SC, Chang EB: Probiotics inhibit nuclear factor-kappaB and induce heat shock proteins in colonic epithelial cells through proteasome inhibition. Gastroenterology 2004, 127:1474-1487

54. Kim JM, Kim JS, Kim YJ, On YK, Kim IY, Chee YJ, Han JS, Jung HC: Conjugated linoleic acids produced by Lactobacillus dissociates IKK-gamma and Hsp90 complex in Helicobacter pylori-infected gastric epithelial cells. Lab Invest 2008, 88:541-552

55. Eun CS, Kim YS, Han DS, Choi JH, Lee AR, Park YK: Lactobacillus casei prevents impaired barrier function in intestinal epithelial cells. APMIS 2011, 119:49-56 